\title{
Migration of Guidewire: A Rare Complication during Arterial Cannulation in a Patient on Intra- arterial Balloon Pump
}

\author{
Sucharita Das ${ }^{1}$ Muralidhar Kanchi ${ }^{1}$ Uday Khanolkar ${ }^{2}$ \\ ${ }^{1}$ Department of Anaesthesia and Critical Care, Narayana Hrudayalaya \\ Institute of Cardiac Sciences, Bengaluru, Karnataka, India \\ 2 Department of Cardiology, Narayana Hrudayalaya Institute of \\ Cardiac Sciences, Bengaluru, Karnataka, India \\ Address for correspondence Dr. Muralidhar Kanchi, MD, FIACTA \\ FICA, MBA, Department of Anaesthesia and Critical Care, Department \\ of Cardiology, Narayana Hrudayalaya Institute of Cardiac Sciences, NH \\ Health city, Bommasandra, Bengaluru 560099, Karnataka, India \\ (e-mail: kanchirulestheworld@gmail.com). \\ J Card Crit Care TSS 2017;1:45-47.
}

\begin{abstract}
Seldinger's technique is frequently used for percutaneous vascular access. This technique has been used for several years successfully for placement of cannulae/

Keywords

- guidewire catheters into the arterial or venous system without the need for cut-down and with

- Seldinger minimal trauma to surrounding structures. In fact, this technique has been adapted to

- intra-aortic balloon introduce angiography catheters, chest tubes, cricothyroidotomy, tracheostomy, pump

- catheter percutaneous endoscopic gastrostomy, etc. Though inherently safe, this technique may be associated with several complications and guidewire-related complication that may be potentially serious, though rare.
\end{abstract}

\section{Introduction}

In $1953,{ }^{1}$ Seldinger described a simple technique for catheter insertion over a guidewire. This is the most commonly used technique to access venous and arterial catheter, hemodialysis catheter, and for chest tube insertion. Guidewire-related complications can be accompanied by significant morbidity and mortality. ${ }^{2}$ In this article, the authors describe a case of "guidewire migration" during femoral artery catheter insertion in a patient who was on intra-arterial balloon pump (IABP) assist device. Subsequently, the patient was taken to catheterization laboratory where fluoroscopy revealed that the guidewire tip was in the carotid artery.

\section{Case Presentation}

A female patient, aged 68 years, known to be suffering from coronary artery disease reported to the authors with recent history of myocardial infarction (MI) and severe left ventricular $(\mathrm{LV})$ dysfunction (ejection fraction $=35 \%$ ). The patient was also known to be suffering with diabetes mellitus and hypothyroidism. While in the hospital, she developed complete heart block for which temporary pacemaker was implanted.
She underwent off-pump coronary artery bypass grafting (CABG) and was shifted from operating theater to the postsurgical intensive care unit with moderate inotropic supports. Next day she became hemodynamically unstable and IABP was inserted to support the cardiovascular system. After 2 days, the patient was tracheostomized as she did not qualify for tracheal extubation. At the same time, left femoral access was secured by Seldinger's technique as no other arterial access was available .While securing the femoral arterial access with a stitch, guidewire was found to be missed. Subsequently, the patient was taken to catheterization laboratory where fluoroscopy revealed that the guidewire tip was in the carotid artery (-Fig. 1). Guidewire was snared out in the same sitting with the help of interventional cardiology (-Fig. 2).

\section{Discussion}

Although in this reported case, the patient did not suffer any consequence of migration of guidewire, guidewire-related complications can significantly increase morbidity and mortality during insertion of invasive catheters. Arterial line placement is a common procedure in various critical care settings as well as during intraoperative major surgeries. It
DOI https://doi.org/ 10.1055/s-0037-1604330.
Copyright $(2017$ Official Publication of License terms The Simulation Society (TSS), accredited by International Society of Cardiovascular Ultrasound (ISCU) 


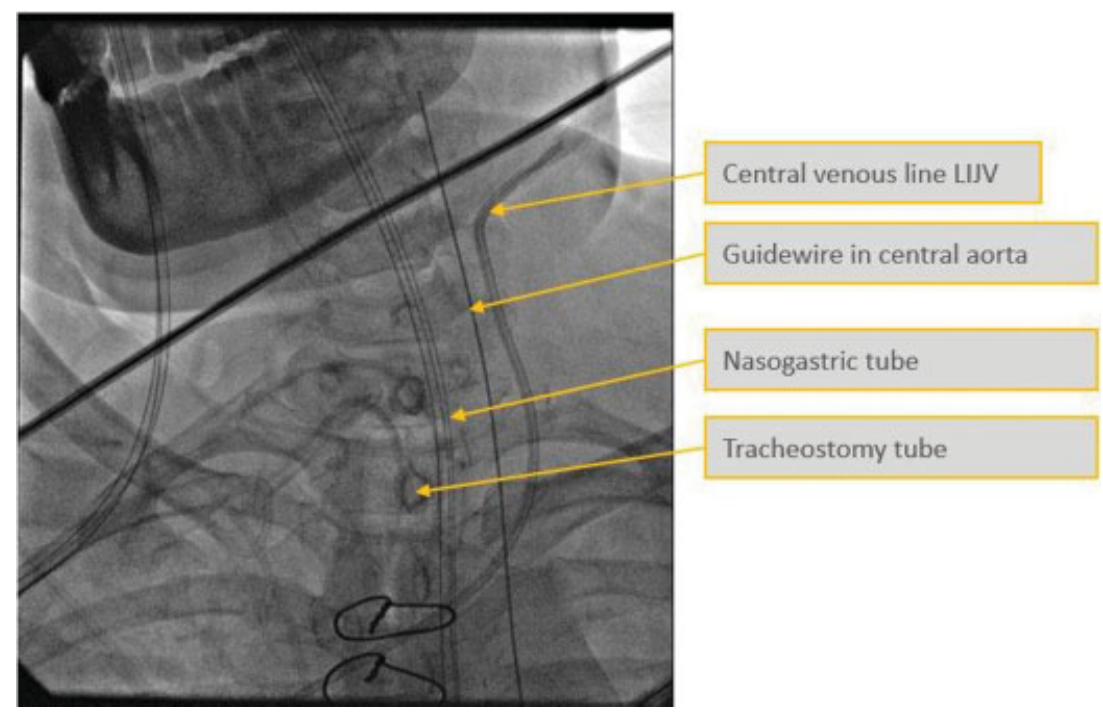

Fig. 1 Snapshot of fluoroscopy picture showing the guidewire in the carotid artery. LIJV, left internal jugular vein.

allows monitoring the rapid recognition of blood pressure changes that is vital for patients on continuous infusion of vasoactive drugs. It is considered a safe procedure with a major complication less than $1 \%{ }^{3}$ The common complications include occlusion, hematoma formation, or infection. Slipping of guidewire into the vessels could be one of avoidable complications.

Intra-aortic balloon pump is a mechanical device that is inserted into descending aorta, just distal to the origin of the left subclavian artery. The basic principle behind the action of intra-aortic balloon pump is known as counter-pulsation. Deflation of the intra-arterial balloon (IAB) occurs in systole. Deflation is typically seen on the screen as being half way down the slope after the dicrotic notch, prior to the atrioventricular (AV) opening. The effect is a decrease in aortic end-diastolic pressure (afterload) by the balloon deflating and creating space in the aorta. The balloon is inflated during diastole, and inflation is timed to coincide with dicrotic notch of arterial waveform. ${ }^{4}$

When the left ventricle contracts, the balloon pump deflates. When the left ventricle relaxes, the balloon pump inflates.
At the onset of ventricular contraction, IABP deflates with the opening of aortic valve. The space occupied by the balloon in the aorta is suddenly released at the onset of ventricular emptying. This causes a vacuum effect or negative pressure in the aorta, thereby reducing ventricular afterload and improving cardiac performance. ${ }^{5}$ Animal research has indicated that during rapid chest compression, intrathoracic pressure can be subatmospheric in some areas of the thorax. ${ }^{6}$ The converse occurs during diastolic inflation of IABP. During diastole, aortic valve gets closed. Inflation of balloon in diastole creates a space-occupying lesion in the aorta displacing blood both proximally and distally. This augments aortic pressure and hence coronary perfusion ( - Fig. 3). A similar case can also be found in the study by Capoccia et al. ${ }^{7}$

The beneficial mechanism of IABP has been proposed to use wave intensity analysis (WIA). ${ }^{6}$ WIA is a method that differentiates between forward running waves propagating in same direction and backward running waves moving in opposite direction. The energy of waves produced by balloon operation has been examined in clinical practice. The energy of backward compressive wave (BCW) produced by balloon

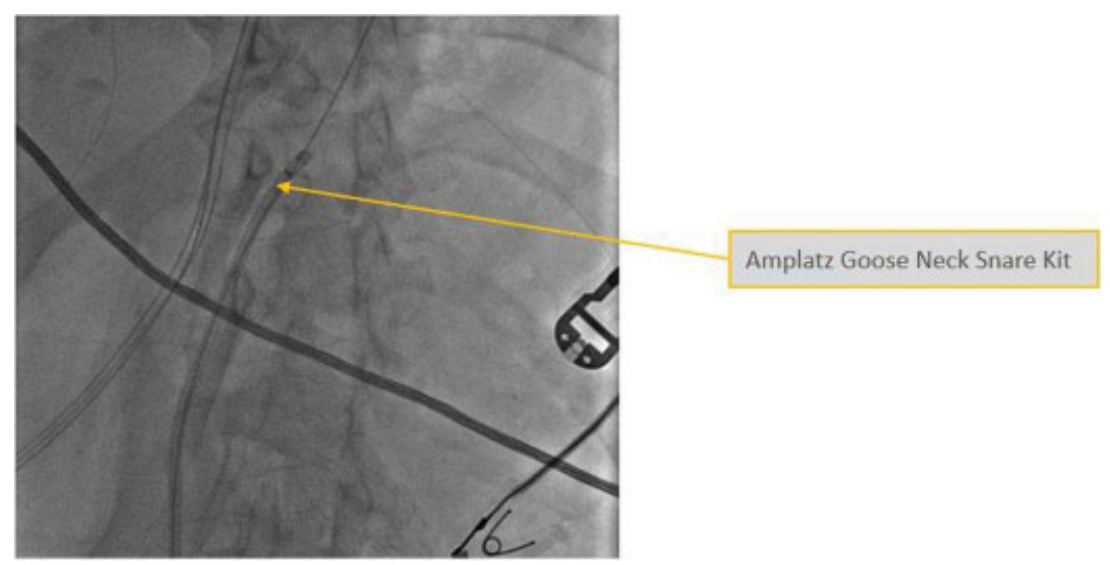

Fig. 2 Snapshot of snare kit. 


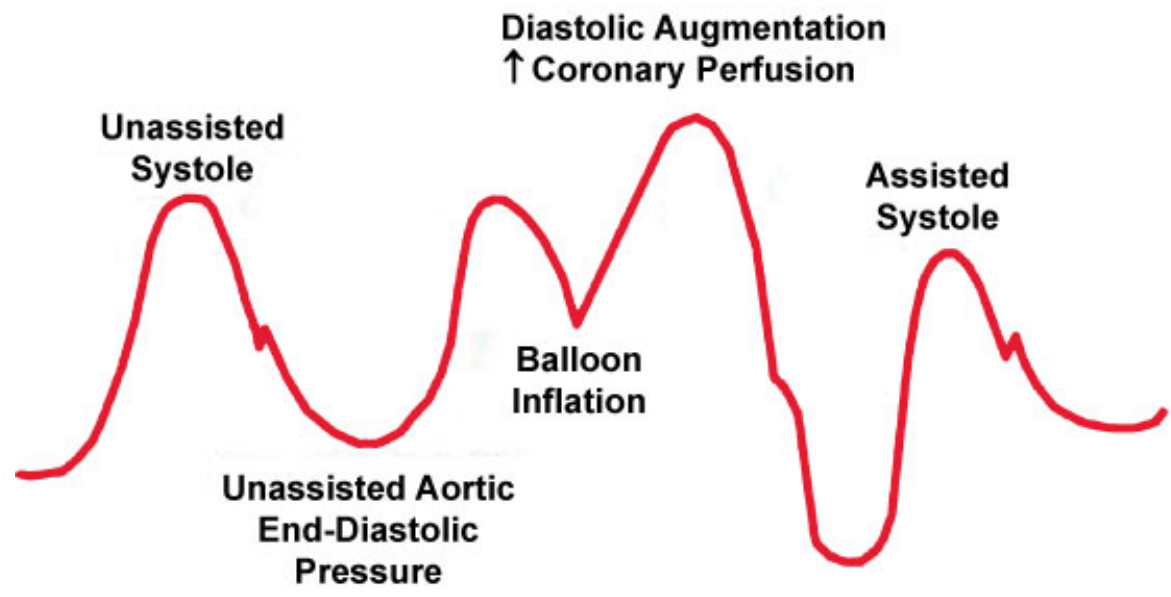

Fig. 3 Intra-arterial balloon pump waveform.

inflation is positively related to pressure augmentation; the backward expansion wave (BEW) generated by negative deflation is correlated to end-diastolic aortic pressure. BCW in balloon inflation generates a pushing effect toward the heart, whereas balloon deflation causes BEW with a pulling effect. The energy of BCW is positively charged and correlates to diastolic aortic pressure augmentation. The energy of BEW is negatively charged and correlates with end-diastolic aortic pressure, which suggests that higher BEW generates lower end-diastolic pressure.

In this case, the length of guidewire was $15 \mathrm{~cm}$ and a length of 3 to $4 \mathrm{~cm}$ was outside the arterial catheter before suturing. While preparing to suture the cannula, guidewire got sucked into the arterial system fast. Later, when patient was taken to cath laboratory, it was found that the catheter tip was in the carotid artery, which suggests that guidewire went up retrograde along the side of balloon, which may be related to negative pressure generated during balloon deflation. Retrograde migrate of guidewire showed that there was a negative pressure that could have sucked the guidewire against the flow.

\section{Conclusion}

To prevent this rare complication, during arterial cannulation via a femoral route using Seldinger's technique on IABP patients, the following preventive measure may be taken:

1. Guidewire should be held from tip all the time during procedure to prevent accidental slipping into the vessels. Insertion of these invasive lines needs some expertise, and guidewire should be immediately taken out as soon as cannula is inserted.
2. The guidewire should not be pushed too far inside the vessel.

3. Before advancing the catheter over guidewire, one must ensure that wire is visible and held at proximal end. If one wishes to keep the guidewire into the vessel before suturing the catheter to the skin, a rubber shot can be used to hold the guidewire. This is more applicable if the patient is on IABP.

4. At the end of procedure, guidewire should be checked in tray.

\section{References}

1 Seldinger SI. Catheter replacement of the needle in percutaneous arteriography; a new technique. Acta Radiol 1953;39(05): 368-376

2 Khasawneh FA, Smalligan RD. Guidewire-related complications during central venous catheter placement: a case report and review of the literature. Case Rep Crit Care 2011;2011:287261

3 Scheer B, Perel A, Pfeiffer UJ. Clinical review: complications and risk factors of peripheral arterial catheters used for haemodynamic monitoring in anaesthesia and intensive care medicine. Crit Care 2002;6(03):199-204

4 Muralidhar K. Intra-aortic balloon pump. In: Cardiac Catheterization and Imaging (From Paediatrics to Geriatrics). New Delhi, India: Jaypee Digital; 2015:chap 68, 1047-1054

5 Paul Collison S, Singh Dagar K. The role of the Intra-aortic balloon pump in supporting children with acute cardiac failure. Postgrad Med J 2007;83(979):308-311

6 Rothman NS, Gelfand M, Burkhoff D, Weisfeldt ML, inventors; Revivant Corporation, assignee. Cardiac assist method using an inflatable vest. United States patent US 6,179,793; January 30, 2001

7 Capoccia M, Bowles CT, Pepper JR, Banner NR, Simon AR. Evidence of clinical efficacy of counterpulsation therapy methods. Heart Fail Rev 2015;20(03):323-335 